TRANSACTIONS OF THE

AMERICAN MATHEMATICAL SOCIETY

Volume 356, Number 3, Pages 987-1005

S 0002-9947(03)03309-9

Article electronically published on October 6, 2003

\title{
SMOOTH SOLUTIONS TO A CLASS OF FREE BOUNDARY PARABOLIC PROBLEMS
}

\author{
OLIVIER BACONNEAU AND ALESSANDRA LUNARDI
}

\begin{abstract}
We establish existence, uniqueness, and regularity results for solutions to a class of free boundary parabolic problems, including the free boundary heat equation which arises in the so-called "focusing problem" in the mathematical theory of combustion. Such solutions are proved to be smooth with respect to time for positive $t$, if the data are smooth.
\end{abstract}

\section{INTRODUCTION}

In this paper we consider the initial value problem for a class of free boundary parabolic equations,

$$
\left\{\begin{array}{l}
u_{t}(t, x)=\mathcal{L} u(t, x)+f(t, x, u(t, x), D u(t, x)), t \geq 0, x \in \Omega_{t}, \\
u(t, x)=g_{0}(t, x), \quad t \geq 0, x \in \partial \Omega_{t} \\
\frac{\partial u}{\partial n}(t, x)=g_{1}(t, x), \quad t \geq 0, x \in \partial \Omega_{t} \\
u(0, x)=u_{0}(x), \quad x \in \Omega_{0}
\end{array}\right.
$$

where the family of open sets $\left\{\Omega_{t}: t \geq 0\right\} \subset \mathbb{R}^{N}$ and the function $u:\{(t, x): t \geq$ $\left.0, x \in \bar{\Omega}_{t}\right\} \mapsto \mathbb{R}$ are the unknowns. By $n=n(t, x)$ we denote the exterior unit normal vector to $\partial \Omega_{t}$ at $x \in \partial \Omega_{t}$.

The data are: the second order elliptic operator $\mathcal{L}=\sum_{i, j=1}^{N} a_{i j}(t, x) D_{i j}$, the functions $f:[0, T] \times \mathbb{R}^{N} \times \mathbb{R} \times \mathbb{R}^{N} \rightarrow \mathbb{R}, g_{0}, g_{1}:[0, T] \times \mathbb{R}^{N} \mapsto \mathbb{R}$, the (possibly unbounded) initial set $\Omega_{0} \subset \mathbb{R}^{N}$, and the initial function $u_{0}: \bar{\Omega}_{0} \mapsto \mathbb{R}$. All of them are assumed to be regular enough. Moreover, $u_{0}$ satisfies the compatibility conditions

$$
u_{0}(x)=g_{0}(x), \quad \frac{\partial u_{0}}{\partial n}(x)=g_{1}(x), \quad x \in \partial \Omega_{0},
$$

and $g_{0}, g_{1}$ satisfy the transversality condition

$$
\frac{\partial g_{0}}{\partial n}(0, x) \neq g_{1}(0, x), \quad x \in \partial \Omega_{0}
$$

Received by the editors May 10, 2001 and, in revised form, July 12, 2002.

2000 Mathematics Subject Classification. Primary 35K05, 35R35.

Key words and phrases. Heat equation, free boundary problems, fully nonlinear parabolic equations.

(C)2003 American Mathematical Society 
In space dimension $N=1$ this problem has been well understood for a long time, see e.g. the review paper [16]. Here we are interested in the multidimensional case $N \geq 2$, where only a few results are available at present.

The simplest and most popular example of (1.1) is the free boundary heat equation,

$$
\left\{\begin{array}{l}
u_{t}=\Delta u \text { in } \Omega_{t}, \quad u=0 \text { and } \frac{\partial u}{\partial n}=C \text { on } \partial \Omega_{t}, \\
u(0, \cdot)=u_{0} \text { in } \Omega_{0},
\end{array}\right.
$$

with $C \neq 0$, motivated by models in combustion theory ([6, 18]) for equidiffusional premixed flames. It can be seen as the high activation energy limit of the regularizing problems $u_{t}=\Delta u-\beta_{\epsilon}(u)$ in $[0, T] \times \mathbb{R}^{N}$, where $\beta_{\epsilon}(s)=\beta_{1}(s / \epsilon) / \epsilon$ has support in a small interval $[-\epsilon, 0]$. In [7, Caffarelli and Vazquez used this regularization to prove existence of global weak solutions to (1.4) for $C^{2}$ initial data $\left(\Omega_{0}, u_{0}\right)$, under suitable geometric assumptions on $u_{0}$. Such solutions may not be unique, and enjoy some regularity properties: the free boundary is locally Lipschitz continuous, and $u$ is $1 / 2$-Hölder continuous with respect to time, Lipschitz continuous with respect to the space variables. The same approach was used in [12] to prove uniqueness of the classical solution to (1.4) in a cylinder for initial data which are monotonic in the direction of the cylinder axis.

In this paper we follow a completely different approach, which leads to existence, uniqueness, and time smoothness for $t>0$ of local regular solutions (with $u$ bounded, in the case of unbounded domains) to problem (1.1). Concerning uniqueness, we prove uniqueness of the solution belonging to a suitable parabolic Hölder space and such that the transversality condition $\frac{\partial g_{0}}{\partial n}(t, x) \neq g_{1}(t, x), x \in \partial \Omega_{t}$, is preserved throughout the interval of existence.

Our approach consists in transforming problem (1.1) into a fully nonlinear parabolic problem in the fixed domain $\Omega_{0}$ for an auxiliary unknown $w$, for which the usual techniques of fully nonlinear parabolic problems in Hölder spaces (see e.g. [14, Ch. 8]) give a local existence and uniqueness result. Coming back to (1.1), for $\Omega_{0}$ with $C^{3+\alpha+k}$ boundary and $u_{0} \in C^{3+\alpha+k}\left(\bar{\Omega}_{0}\right)$ we obtain a local solution with $\partial \Omega_{t} \in C^{2+\alpha+k}$ and $u(t, \cdot)$ in $C^{2+\alpha+k}\left(\bar{\Omega}_{t}\right), k=0,1$. So, we share with [7] the unpleasant fact that we lose one degree of space regularity for $t>0$. Although this is natural for global weak solutions, as simple examples described in $[7$ show, it is not wholly satisfactory for local classical solutions. One could expect that in a small time interval the solution remains at least as regular as the initial datum: this is what happens in other free boundary problems of parabolic type (see e.g. 8, 9 for boundary conditions of Stefan type), and in problem (1.1) for special initial data. Thus, our main results are uniqueness and time smoothness, and we consider our existence theorem just as a tool to prove the other results.

A noteworthy situation in which there is no loss of regularity, at least in small time intervals, is the case of initial data near special smooth solutions, such as stationary solutions, self-similar solutions, travelling waves. In particular, $C^{2+\alpha}$ initial data near any regular stationary solution $(\Omega, U)$ with bounded $\Omega$ were considered in the paper [3], where we studied stability of regular stationary solutions, establishing a linearized stability principle for (1.1) in the time-independent case. Since self-similar solutions to (1.4) become stationary solutions to a problem of the type (1.1) after a suitable change of coordinates, we could also consider initial data for 
(1.4) near self-similar solutions. In the unbounded domain case, $C^{2+\alpha}$ initial data near a planar travelling wave solution of (1.4) have been considered in [5]. The wave turns out to be orbitally stable, but the discussion is not trivial, because in dimension $N \geq 2$ this is a very critical case of stability.

Another example without loss of regularity was considered in [1, where a twophase version of (1.1) was studied in a cylinder for $C^{2+\alpha}$ initial data far from special solutions but satisfying suitable monotonicity conditions: $u_{0}$ is assumed to be strictly monotonic in the direction orthogonal to the cylinder axis. This allows one to take $u$ as a new independent variable for small $t$, an old trick already used by Meirmanov in the Stefan problem and also in a two-dimensional version of problem (1.1), see [15].

Together with loss of regularity, the other big question about problem (1.1) is uniqueness of the classical solution. Indeed, the uniqueness results available up to now concern only particular situations, such as radially symmetric solutions of (1.4), studied in [10], and solutions in cylinders, for initial data which are monotonic in the direction of the axis of the cylinder (see [15] in dimension $N=2$ and [12] in any dimension), or in the direction orthogonal to the axis of the cylinder (see 1], for the two-phase case). Moreover, the above mentioned papers [3, 5] also give uniqueness results in parabolic Hölder spaces, but only for solutions close to the special solutions considered.

Therefore, the problem of wellposedness for general initial data in dimension bigger than 1 still remains open, even in the simplest case (1.4).

Below we briefly describe our approach, which is similar to the approach of [3].

We look for $\partial \Omega_{t}$ as the range of the function $\xi \mapsto \xi+s(t, \xi) \nu(\xi)$, defined for $\xi \in \partial \Omega_{0}$, where $\nu(\xi)$ is the exterior unit normal vector to $\partial \Omega_{0}$ at $\xi$, and the unknown $s(t, \xi)$ is the signed distance of the point $x=\xi+s(t, \xi) \nu(\xi)$ from $\partial \Omega_{t}$. Then by a natural change of coordinates we transform $\Omega_{t}$ into $\Omega_{0}$, at least for small time, and denoting by $\widetilde{u}$ the unknown $u$ expressed in the new coordinates, we get a fixed boundary system for $(s, \widetilde{u})$, in the domain $[0, \delta] \times \bar{\Omega}_{0}$ with small $\delta$. The change of coordinates is defined by $\xi \mapsto \xi+s(t, \xi) \nu(\xi)$ for $\xi \in \partial \Omega_{0}$, and it is extended smoothly to a $C^{2+\alpha}$ diffeomorphism $\xi \mapsto \xi+\Phi(t, \xi)$ in the whole of $\Omega_{0}$.

The main step is now the introduction of a new unknown $w$ defined by

$$
\widetilde{u}(t, \xi)=u_{0}(\xi)+\left\langle D u_{0}(\xi), \Phi(t, \xi)\right\rangle+w(t, \xi),
$$

which allows us to decouple the system using the boundary condition $u=g_{0}$ at $\partial \Omega_{t}$. In the case $g_{0} \equiv 0, g_{1} \equiv C$ we get simply

$$
s(t, \xi)=-\frac{w(t, \xi)}{C}, \quad t \geq 0, \xi \in \partial \Omega_{0} .
$$

In the general case the formula is a bit more complicated. It comes out

$$
s(t, \xi)=\frac{w(t, \xi)}{\frac{\partial g_{0}}{\partial \nu}(t, \xi)-g_{1}(0, \xi)}+R(t, \xi, w(t, \xi)),
$$

where the remainder $R(t, \xi, u)$ vanishes at $(0, \xi, 0)$ together with $R_{u}$. It is here that we need the transversality assumption (1.3). 
In any case, we can go through, and we get a final problem for the only unknown $w$,

$$
\left\{\begin{array}{l}
w_{t}=\mathcal{A} w+F(t, w), \quad t \geq 0, \xi \in \bar{\Omega}_{0}, \\
\mathcal{B} w=G(t, w), \quad t \geq 0, \xi \in \partial \Omega_{0}, \\
w(0, \cdot)=0, \quad \xi \in \bar{\Omega}_{0},
\end{array}\right.
$$

where $\mathcal{A}, \mathcal{B}$ are linear differential operators and $F, G$ are nonlinear functions. Problem (1.6) is fully nonlinear, because $F$ depends on $w$ and on its space derivatives up to the second order, and $G$ depends on $w$ and on its first order space derivatives. Moreover, $F$ is nonlocal in the second order derivatives of $w$. However, both $F$ and $G$ are smooth enough, and $F_{w}, G_{w}$ vanish at $t=0, w=0$. The boundary operator $\mathcal{B}$ is obtained from the Neumann boundary condition $\frac{\partial u}{\partial n}=g_{1}$ after the change of coordinates and unknowns; in the case $g_{0} \equiv 0, g_{1} \equiv C$ we get

$$
\mathcal{B} v=\frac{\partial v}{\partial \nu}-\frac{1}{C} \frac{\partial^{2} u_{0}}{\partial \nu^{2}} v
$$

The second order linear operator $\mathcal{A}$ is the sum of $\mathcal{L}$ plus a nonlocal operator, acting only on $w$ and on the first order space derivatives of $w$ at $t=0$. This is crucial for our analysis: since the principal part of $\mathcal{A}$ coincides with the principal part of $\mathcal{L}$ at $t=0$, the linearized problem near $t=0, w_{0}=0$ is a good linear parabolic problem, for which optimal Hölder regularity results and estimates are available; we need such estimates to solve the nonlinear problem in a standard way, using the contraction theorem in a ball of the parabolic Hölder space $C^{1+\alpha / 2,2+\alpha}\left([0, \delta] \times \bar{\Omega}_{0}\right)$, with $\delta>0$ suitably small, or else in a ball of $C^{3 / 2+\alpha / 2,3+\alpha}\left([0, \delta] \times \bar{\Omega}_{0}\right)$ if the data are more regular.

It is clear now why we lose regularity: not only because of the change of coordinates, but also because $w$ has the same space regularity of $D u_{0}$, due to the splitting (1.5). This problem may be avoided for initial data near smooth (at least $C^{3+\alpha}$ ) stationary solutions, where it is possible to linearize around the stationary solution itself; see [3].

However, it is easy to see that uniqueness of $w$ implies uniqueness of $(s, \widetilde{u})$, and hence uniqueness of the regular solution to the original problem (1.1). Moreover, using standard techniques of fully nonlinear problems, it is possible to prove that $t \mapsto w(t, \cdot)$ is analytic with values in $C^{2}\left(\bar{\Omega}_{0}\right)$ in the interval $(0, \delta)$ if the data are analytic. It follows that both the free boundary and $u$ are analytic with respect to time for $t$ in $(0, \delta)$.

By a covering argument we get eventually that the free boundary of any sufficiently smooth solution defined in an interval $[0, a]$ is time analytic in $(0, a]$, provided $\frac{\partial g_{0}}{\partial n}(t, x) \neq g_{1}(t, x)$ for $0 \leq t \leq a, x \in \partial \Omega_{t}$.

\section{TRANSFORMATION TO A FULLY NONLINEAR FIXED BOUNDARY PROBLEM}

We write below the precise regularity assumption on the data. We fix once and for all a number $\alpha \in(0,1)$.

(a) The initial data. The initial data are couples $\left(\Omega_{0}, u_{0}\right)$, where $\Omega_{0} \subset \mathbb{R}^{N}$ is a nonempty open set with $C^{3+\alpha}$ boundary $\partial \Omega_{0}$. If $\Omega_{0}$ is unbounded, we need that the boundary is uniformly $C^{3+\alpha}$, in the sense that the diffeomorphisms which locally straighten the boundary and their inverses have $C^{3+\alpha}$ norms bounded by a 
constant. Moreover, $u_{0} \in C^{3+\alpha}\left(\bar{\Omega}_{0}\right)$. In particular, $u_{0}$ and all its partial derivatives up to the third order are bounded.

(b) The other data. We have

$$
\mathcal{L} v=a_{i j}(t, x) D_{i j} v
$$

where the coefficients $a_{i j}$ and their first order space derivatives belong to the space $C^{\alpha / 2, \alpha}\left([0, T] \times \mathbb{R}^{N}\right)$, and satisfy a uniform ellipticity condition: there is $\nu>0$ such that $a_{i j}(t, x) \xi_{i} \xi_{j} \geq \nu|\xi|^{2}$ for all $t, x, \xi$.

The function $f=f(t, x, u, p)$ is once differentiable with respect to $x$, twice differentiable with respect to $(u, p) . f$ and its derivatives are continuous in $(t, x, u, p)$, and $C^{\alpha / 2, \alpha}$ in $(t, x)$, locally uniformly with respect to $(u, p)$.

Moreover, $g_{0}$ and $g_{1}$ are differentiable with respect to the space variables, and $g_{0}, D_{i} g_{0} \in C^{1+\alpha / 2,2+\alpha}\left([0, T] \times \mathbb{R}^{N}\right), g_{1}, D_{i} g_{1} \in C^{1 / 2+\alpha / 2,1+\alpha}\left([0, T] \times \mathbb{R}^{N}\right)$ for $i=1, \ldots, N$.

(c) Compatibility and transversality conditions. Since we deal with regular solutions up to $t=0$, we have to assume that (1.2) holds. We assume furthermore that (1.3) holds. This is a condition on the structure of the problem; it says that ours is not an obstacle-like problem. However, it is necessary for our approach to work.

2.1. The change of coordinates. For every $\xi \in \partial \Omega_{0}$ let $\nu(\xi)=n(0, \xi)$ be the unit exterior normal vector to $\partial \Omega_{0}$. There is a neighborhood $\mathcal{N}$ of $\partial \Omega_{0}$ such that each $x \in \mathcal{N}$ may be written in a unique way as $x=\xi+s \nu(\xi)$ with $\xi \in \partial \Omega_{0}$ and $s \in \mathbb{R}$. The number $s$ is called the signed distance of $x$ from $\partial \Omega_{0}$, and it is a regular function of $x$. As we consider local regular solutions, for $t$ small enough $\partial \Omega_{t} \subset \mathcal{N}$; we shall represent $\partial \Omega_{t}$ as

$$
\partial \Omega_{t}=\left\{\xi+s(t, \xi) \nu(\xi) ; \xi \in \partial \Omega_{0}\right\},
$$

where $s(t, \xi)$ is an unknown regular function. The function $\xi \mapsto \xi+s(t, \xi) \nu(\xi)$ is a diffeomorphism from $\partial \Omega_{0}$ to $\partial \Omega_{t}$, and it may be extended in a standard way to a diffeomorphism from $\Omega_{0}$ to $\Omega_{t}$, as follows. First we extend the normal field: for every $\xi \in \mathcal{N}$ we denote by $\xi^{\prime}$ the point of $\partial \Omega_{0}$ closest to $\xi$, and then for every $\xi \in \mathbb{R}^{N}$ we set

$$
\widetilde{\nu}(\xi)=\left\{\begin{array}{l}
\theta(\xi) \nu\left(\xi^{\prime}\right) \text { if } \xi \in \mathcal{N}, \\
0 \text { otherwise }
\end{array}\right.
$$

where $\theta$ is a $C^{\infty}$ function with support contained in $\mathcal{N}$, such that $\theta \equiv 1$ in a neighborhood of $\partial \Omega_{0}$ and $\theta \equiv 0$ outside a bigger neighborhood of $\partial \Omega_{0}$.

Then we set

$$
\Phi(t, \xi)=\left\{\begin{array}{l}
\theta(\xi) s\left(t, \xi^{\prime}\right) \nu\left(\xi^{\prime}\right) \quad \text { if } \xi \in \mathcal{N}, \\
0 \text { otherwise. }
\end{array}\right.
$$

To simplify formulas, with a slight abuse of notation we shall write

$$
\Phi(t, \xi)=s\left(t, \xi^{\prime}\right) \widetilde{\nu}(\xi) .
$$

More generally, if $a$ is a function defined in $[0, T] \times \mathcal{N} \cap \bar{\Omega}_{0}$ and $b$ is a function defined in $\bar{\Omega}_{0}$, with support contained in $\mathcal{N} \cap \bar{\Omega}_{0}$, we shall write $a \cdot b$ to denote the function $(t, \xi) \mapsto a(t, \xi) b(\xi)$ for $\xi \in \mathcal{N} \cap \bar{\Omega}_{0},(t, \xi) \mapsto 0$ if $\xi \in \bar{\Omega}_{0} \backslash \mathcal{N}$. 
For $t$ small, the mapping

$$
\xi \mapsto x(t, \xi)=\xi+\Phi(t, \xi), \quad \xi \in \mathbb{R}^{N},
$$

is then a $C^{2+\alpha}$ diffeomorphism from $\mathbb{R}^{N}$ to itself, which maps $\Omega_{0}$ onto $\Omega_{t}$.

Let $\widetilde{u}$ denote the unknown $u$ in the variables $(t, \xi)$, i.e.

$$
\widetilde{u}(t, \xi)=u(t, \xi+\Phi(t, \xi)), \quad t \geq 0, \xi \in \bar{\Omega}_{0} .
$$

Similarly, we set $\widetilde{a}_{i j}(t, \xi)=a_{i j}(t, \xi+\Phi(t, \xi))$.

With the change of variables $(t, x) \mapsto(t, \xi)$, the equation $u_{t}=\mathcal{L} u+f$ is transformed to

$\widetilde{u}_{t}-\left\langle D \widetilde{u},\left(I+{ }^{t} D \Phi\right)^{-1} \Phi_{t}\right\rangle=\widetilde{\mathcal{L}} \widetilde{u}+f\left(t, \xi+\Phi(t, \xi), \widetilde{u},\left(I+{ }^{t} D \Phi\right)^{-1} D \widetilde{u}\right), \quad t \geq 0, \xi \in \bar{\Omega}_{0}$, where $[D \Phi]_{i j}=\frac{\partial \Phi_{i}}{\partial \xi_{j}}(t, \xi)$, the superscript $t$ denotes the transposed matrix, and $\widetilde{\mathcal{L}}$ is the new expression of the operator $\mathcal{L}$, i.e.

$$
\widetilde{\mathcal{L}}=\sum_{i, j, h, k=1}^{N} \widetilde{a}_{i k} \frac{\partial \xi_{j}}{\partial x_{i}} \frac{\partial \xi_{h}}{\partial x_{k}} D_{j h}+\sum_{i, j, k=1}^{N} \widetilde{a}_{i k} \frac{\partial^{2} \xi_{j}}{\partial x_{i} \partial x_{k}} D_{j}
$$

Here for each $t$ the function $\xi(t, x)$ is the inverse of (2.4), so that the matrix with components $\frac{\partial \xi_{h}}{\partial x_{k}}$ is nothing but $(I+D \Phi(t, \xi))^{-1}$.

2.2. The splitting. To decouple problem (2.6) we introduce a new unknown $w$ defined by

$$
\widetilde{u}(t, \xi)=u_{0}(\xi)+\left\langle D u_{0}(\xi), \Phi(t, \xi)\right\rangle+w(t, \xi), t \geq 0, \xi \in \bar{\Omega}_{0} .
$$

Accordingly, we rewrite (2.6) as

$$
\begin{aligned}
& \left\langle D u_{0}, \Phi_{t}\right\rangle+w_{t}-\left\langle D\left(u_{0}+\left\langle D u_{0}, \Phi\right\rangle+w\right),\left(I+{ }^{t} D \Phi\right)^{-1} \Phi_{t}\right\rangle \\
& =\widetilde{\mathcal{L}}\left(u_{0}+\left\langle D u_{0}, \Phi\right\rangle+w\right) \\
& \quad+f\left(t, \xi+\Phi, u_{0}+\left\langle D u_{0}, \Phi\right\rangle+w,\left(I+{ }^{t} D \Phi\right)^{-1} D\left(u_{0}+\left\langle D u_{0}, \Phi\right\rangle+w\right)\right), \\
& \quad t \geq 0, \xi \in \bar{\Omega}_{0},
\end{aligned}
$$

The decomposition (2.7) may look a bit strange; it is similar to the decompositions used in the papers [3, 4, 5] and in others to study stability problems. It is crucial in our analysis because it lets us decouple system (2.8), expressing $s$ in terms of $w$. Indeed, the boundary condition $u-g_{0}=0$ on $\partial \Omega_{t}$ is rewritten as

$$
g_{0}(0, \xi)+s(t, \xi) g_{1}(0, \xi)+w(t, \xi)-g_{0}(t, \xi+s(t, \xi) \nu(\xi))=0, \quad \xi \in \partial \Omega_{0},
$$

that is, $F(t, \xi, s, w)=0$, with $F_{s}(t, \xi, s, w)=g_{1}(0, \xi)-\frac{\partial g_{0}}{\partial \nu}(t, \xi) \neq 0$ for $t$ small, due to the transversality assumption (1.3). By the implicit function theorem we get $s$ as a function of $(t, \xi, w)$ for small $w$; more precisely,

$$
s(t, \xi)=\mathcal{F}_{0}(t, \xi, w(t, \xi)), \quad t \geq 0, \xi \in \partial \Omega_{0},
$$

so that

$$
\Phi(t, \xi)=\mathcal{F}_{0}\left(t, \xi^{\prime}, w\left(t, \xi^{\prime}\right)\right) \widetilde{\nu}(\xi), \quad t \geq 0, \xi \in \bar{\Omega}_{0} .
$$

Note that in the free boundary heat equation (1.4) formula (2.10) reduces to

$$
s(t, \xi)=-\frac{w(t, \xi)}{C}, t \geq 0, \xi \in \bar{\Omega}_{0} .
$$


It is clear now why we need the transversality assumption (1.3); it is used to get the unknown $s$ in terms of $w$, decoupling the system.

For future use we remark here that for each $\xi \in \partial \Omega_{0}$ we have

$\mathcal{F}_{0}(t, \xi, w(t, \xi))=\frac{w(t, \xi)-g_{0}(t, \xi)+g_{0}(0, \xi)}{\frac{\partial g_{0}}{\partial \nu}(t, \xi)-g_{1}(0, \xi)}+Q\left(t, \xi, w(t, \xi)-g_{0}(t, \xi)+g_{0}(0, \xi)\right)$,

where $Q(t, \xi, 0)=Q_{u}(t, \xi, 0)=0$.

Substituting (2.11) in (2.8), we get

$$
w_{t}=\mathcal{F}_{1}\left(t, \xi, w, D w, D^{2} w\right)+\mathcal{F}_{2}(t, \xi, w, D w) s_{t}, \quad t \geq 0, \xi \in \bar{\Omega}_{0},
$$

where $\mathcal{F}_{1}, \mathcal{F}_{2}$ are obtained respectively from

$\widetilde{\mathcal{L}}\left(u_{0}+\left\langle D u_{0}, \Phi\right\rangle+w\right)+f\left(t, \xi+\Phi, u_{0}+\left\langle D u_{0}, \Phi\right\rangle+w,\left(I+{ }^{t} D \Phi\right)^{-1} D\left(u_{0}+\left\langle D u_{0}, \Phi\right\rangle+w\right)\right)$

and from

$$
-\left\langle D u_{0}-(I+D \Phi)^{-1} D\left(u_{0}+\left\langle D u_{0}, \Phi\right\rangle+w\right), \widetilde{\nu}\right\rangle,
$$

substituting $\Phi=\mathcal{F}_{0}\left(t, \xi^{\prime}, w\left(t, \xi^{\prime}\right)\right) \widetilde{\nu}(\xi)$.

Equation (2.13) still contains $s_{t}$; to eliminate it we again use the identity (2.9), which gives, after differentiation with respect to time,

$$
w_{t}=\frac{\partial g_{0}}{\partial t}(t, \xi+s \nu)+\left(\left\langle D g_{0}(t, \xi+s \nu), \nu\right\rangle-g_{1}(0, \xi)\right) s_{t}, \quad t \geq 0, \xi \in \partial \Omega_{0} .
$$

Substituting in (2.13) for $\xi \in \partial \Omega_{0}$, we get

$$
\begin{aligned}
s_{t}\left(\left\langle D g_{0}\left(t, \xi+\mathcal{F}_{0}(t, \xi, w) \nu\right), \nu\right\rangle-g_{1}(0, \xi)-\mathcal{F}_{2}(t, \xi, w, D w)\right) \\
\quad+\frac{\partial g_{0}}{\partial t}\left(t, \xi+\mathcal{F}_{0}(t, \xi, w) \nu\right) \\
=\mathcal{F}_{1}\left(t, \xi, w, D w, D^{2} w\right), \quad t \geq 0, \xi \in \partial \Omega_{0},
\end{aligned}
$$

so that, at least for $t$ small, it is possible to get $s_{t}$ as a function of $w$,

$$
s_{t}=\mathcal{F}_{3}\left(t, \xi, w, D w, D^{2} w\right)=\frac{\mathcal{F}_{1}-\frac{\partial g_{0}}{\partial t}\left(t, \xi+\mathcal{F}_{0} \nu\right)}{\left\langle D g_{0}\left(t, \xi+\mathcal{F}_{0} \nu\right), \nu\right\rangle-g_{1}(0, \xi)-\mathcal{F}_{2}},
$$

which, substituted in (2.13), gives the final equation for $w$,

$$
w_{t}=\mathcal{F}(t, w)(\xi), \quad t \geq 0, \xi \in \bar{\Omega}_{0},
$$

where

$$
\mathcal{F}(t, w)(\xi)=\mathcal{F}_{1}\left(t, \xi, w, D w, D^{2} w\right)+\mathcal{F}_{2}(t, \xi, w, D w) \mathcal{F}_{3}\left(t, \xi, w, D w, D^{2} w\right) .
$$

Although the explicit expression of $\mathcal{F}$ is rather complicated, we may note immediately that for $\xi$ in $\Omega_{0} \backslash \mathcal{N}$, that is far from the boundary, we have

$$
\mathcal{F}(t, w)(\xi)=\mathcal{L} w+\mathcal{L} u_{0}+f\left(t, \xi, w+u_{0}, D w+D u_{0}\right) .
$$

This is due to the fact that our change of coordinates reduces to the identity far from the boundary. Moreover, even near the boundary we have

$$
\mathcal{F}(0,0)(\xi)=\sum_{i, j=1}^{N} a_{i j}(0, \xi) D_{i j} u_{0}(\xi)+f\left(0, \xi, u_{0}(\xi), D u_{0}(\xi)\right) .
$$


For $\xi$ near the boundary, the function $\mathcal{F}(t, v)$ is defined for $t$ small and for $v \in$ $C^{2}\left(\bar{\Omega}_{0}\right)$ with small $C^{1}$ norm. More precisely, let $\delta_{0} \in(0, T]$ be such that

$$
\frac{\partial g_{0}}{\partial \nu}(t, \xi)-g_{1}(0, \xi) \neq 0,0 \leq t \leq \delta_{0}, \xi \in \partial \Omega_{0} .
$$

The function $\mathcal{F}(t, v)$ is defined for $t \in\left[0, \delta_{0}\right]$, and for $v \in C^{2}\left(\bar{\Omega}_{0}\right)$ with $\|v\|_{C^{1}} \leq r$, in such a way that both $\mathcal{F}_{0}(t, \xi, v(\xi))$ and $\mathcal{F}_{3}\left(t, \xi, v(\xi), D v(\xi), D^{2} v(\xi)\right)$ are well defined for $\xi \in \partial \Omega_{0}$ and for $\xi \in \bar{\Omega}_{0}$, respectively.

From formulas (2.10), 2.14), 2.15), 2.16) we see that $\mathcal{F}(t, v)(\xi)$ depends on $v, D v, D^{2} v$ and their traces at the boundary through functions which are at least $C^{\alpha / 2, \alpha}$ with respect to $(t, \xi)$ and at least $C^{2+\alpha}$ with respect to $v$ and to its space derivatives of the first and second orders. Therefore, for $0 \leq t \leq \delta_{0}$, the function $v \mapsto \mathcal{F}(t, v)$ is continuously differentiable from $\left\{v \in C^{2}\left(\bar{\Omega}_{0}\right):\|v\|_{C^{1}} \leq r\right\}$ to $C\left(\bar{\Omega}_{0}\right)$, and from $\left\{v \in C^{2+\alpha}\left(\bar{\Omega}_{0}\right):\|v\|_{C^{1}} \leq r\right\}$ to $C^{\alpha}\left(\bar{\Omega}_{0}\right)$.

In the next lemma we describe the linear part of $\mathcal{F}$ with respect to $v$ near $v=0$. We recall that for every $\xi \in \mathcal{N}, \xi^{\prime}$ denotes the point of $\partial \Omega_{0}$ closest to $\xi$.

Lemma 2.1. For every $t \in\left[0, \delta_{0}\right]$ we have $\mathcal{F}_{v}(t, 0)=\mathcal{A}(t, \partial)$, where

$$
\begin{aligned}
(\mathcal{A}(t, \partial) v)(\xi)= & \sum_{i, j=1}^{n}\left(\alpha_{i j}(t, \xi) D_{i j} v(\xi)+\alpha_{i j}^{\prime}(t, \xi) D_{i j} v\left(\xi^{\prime}\right)\right) \\
& +\sum_{i=1}^{n}\left(\beta_{i}(t, \xi) D_{i} v(\xi)+\beta_{i}^{\prime}(t, \xi) D_{i} v\left(\xi^{\prime}\right)\right) \\
& +\gamma(t, \xi) v(\xi)+\gamma^{\prime}(t, \xi) v\left(\xi^{\prime}\right), 0 \leq t \leq T, \quad \xi \in \bar{\Omega}_{0} .
\end{aligned}
$$

The coefficients $\alpha_{i j}, \beta_{i}, \gamma, \alpha_{i j}^{\prime}, \beta_{i}^{\prime}, \gamma^{\prime}$ are in $C^{\alpha / 2, \alpha}\left(\left[0, \delta_{0}\right] \times \bar{\Omega}_{0}\right) . \alpha_{i j}^{\prime}, \beta_{i}^{\prime}, \gamma^{\prime}$ vanish in $\Omega_{0} \backslash \mathcal{N}$, and

$$
\alpha_{i j}(0, \xi)=a_{i j}(0, \xi), \quad \alpha_{i j}^{\prime}(0, \xi)=0, \quad \xi \in \bar{\Omega}_{0} .
$$

Proof. It is clear from the expression of $\mathcal{F}$ that the linear part of $v \mapsto \mathcal{F}(t, v)$ near $v=0$ is a nonlocal second order linear differential operator $\mathcal{A}(t, \partial)$ of the type (2.20). To identify its principal part at $t=0$, it is sufficient to consider $\mathcal{F}_{1}$. Indeed, the product $\mathcal{F}_{2}(0, \xi, v, D v) \mathcal{F}_{3}\left(0, \xi, v, D v, D^{2} v\right)$ does not contribute to the principal part, because $\mathcal{F}_{2}$ does not depend on the second order derivatives of $v$, and it vanishes at $t=0, v=0$.

Using (2.14), we see that only the term $\widetilde{\mathcal{L}}\left(u_{0}+\left\langle D u_{0}, \Phi\right\rangle+w\right)$ in $\mathcal{F}_{1}$ contributes to the principal part of $\mathcal{A}(0, \partial)$. Its explicit expression is

$$
\begin{aligned}
& \sum_{i, k, h, j=1}^{N} a_{i k}(0, \xi+\Phi) \frac{\partial \xi_{j}}{\partial x_{i}}(\xi+\Phi) \frac{\partial \xi_{h}}{\partial x_{k}}(\xi+\Phi) D_{j h}\left(u_{0}+\left\langle D u_{0}, \Phi\right\rangle+v\right) \\
& +\sum_{i, k, j=1}^{N} a_{i k}(0, \xi+\Phi) \frac{\partial^{2} \xi_{j}}{\partial x_{i} \partial x_{k}}(\xi+\Phi) D_{j}\left(u_{0}+\left\langle D u_{0}, \Phi\right\rangle+v\right) \\
& =\mathcal{L}_{0}(v, \Phi)+\mathcal{L}_{1}(v, \Phi)
\end{aligned}
$$

where the matrix $M$ with entries $m_{h k}=\partial \xi_{h} / \partial x_{k}$ is equal to $(I+D \Phi)^{-1}$. We recall that $\Phi$ depends on $v$ through (2.10) and (2.3), i.e. $\Phi(t, \xi, v)=\mathcal{F}_{0}\left(t, \xi^{\prime}, v\left(\xi^{\prime}\right)\right) \widetilde{\nu}(\xi)$ and $\Phi(0, \xi, 0)=0$. 
At $t=0, v=0, \Phi=0$, the linear part of $\mathcal{L}_{0}$ with respect to $(v, \Phi)$ is

$$
\sum_{i, k=1}^{N} a_{i k}(0, \xi)\left(D_{i k} v+D_{i k}\left\langle D u_{0}, \Phi\right\rangle\right)+\text { lower order terms. }
$$

From the developement

$$
(I+D \Phi)^{-1}=I-D \Phi+(D \Phi)^{2}(I+D \Phi)^{-1}
$$

we get that the linear part of $\mathcal{L}_{1}$ with respect to $(v, \Phi)$ is

$$
-\sum_{i, k, j=1}^{N} a_{i k}(0, \xi) \frac{\partial^{2} \Phi_{j}}{\partial x_{i} \partial x_{k}} D_{j} u_{0}+\text { lower order terms. }
$$

Therefore the second order derivatives of $\Phi$ cancel in the sum; what remains is

$$
\mathcal{A}(0, \partial)=\sum_{i, k=1}^{N} a_{i k}(0, \xi) D_{i k}+\text { lower order terms, }
$$

and the statement follows.

Lemma 2.1 implies that equation (2.17) is parabolic near $t=0$.

Equation (2.17) has to be supported with an initial and a boundary condition. The initial condition comes from the splitting (2.7); it is simply

$$
w(0, \xi)=0, \quad \xi \in \bar{\Omega}_{0} .
$$

The boundary condition arises from $\partial u / \partial n=g_{1}$. Since

$$
n(t, \xi+\Phi)=\frac{\left(I+{ }^{t} D \Phi\right)^{-1} \nu(\xi)}{\left|\left(I+{ }^{t} D \Phi\right)^{-1} \nu(\xi)\right|}
$$

and

$$
D u(t, \xi+\Phi)=\left(I+{ }^{t} D \Phi\right)^{-1} D \widetilde{u}(t, \xi),
$$

from $\partial u / \partial n=g_{1}$ we get

$\left\langle\left(I+{ }^{t} D \Phi\right)^{-1} \nu,\left(I+{ }^{t} D \Phi\right)^{-1} D\left(u_{0}+\left\langle D u_{0}, \Phi\right\rangle+w\right)\right\rangle-g_{1}(t, \xi+\Phi)\left|\left(I+{ }^{t} D \Phi\right)^{-1} \nu\right|=0$,

which gives a new boundary condition for $w$,

$$
\mathcal{G}(t, \xi, w(t, \xi), D w(t, \xi))=0, \quad t \geq 0, \xi \in \partial \Omega_{0},
$$

as soon as we substitute $\Phi=\mathcal{F}_{0}\left(t, \xi^{\prime}, w\left(t, \xi^{\prime}\right)\right) \widetilde{\nu}(\xi)$ in (2.22). The function $\mathcal{G}(t, \xi, u, p)$ and its first and second order derivatives with respect to $u$ and $p_{i}, i=1, \ldots, N$, are continuous in $(t, \xi, u, p)$ and $C^{1 / 2+\alpha / 2,1+\alpha}$ in $(t, \xi)$. Moreover, $\mathcal{G}(0, \xi, 0,0)=0$.

In the next lemma we identify the linear part of $\mathcal{G}$ with respect to $(u, p)$ at $(0, \xi, 0,0)$.

Lemma 2.2. We have

$$
\mathcal{G}(t, \xi, w, D w)=\mathcal{B} w-G(t, \xi, w, D w)(\xi),
$$

where $\mathcal{B}$ is the linear differential operator defined by

$$
\begin{aligned}
\mathcal{B} v= & \frac{\partial v}{\partial \nu}+v\left(\frac{\frac{\partial^{2} u_{0}}{\partial \nu^{2}}(\xi)-\frac{\partial g_{1}}{\partial \nu}(0, \xi)}{\frac{\partial g_{0}}{\partial \nu}(0, \xi)-g_{1}(0, \xi)}\right) \\
& -\left\langle D^{\operatorname{tang}}\left(\frac{v}{\frac{\partial g_{0}}{\partial \nu}(0, \xi)-g_{1}(0, \xi)}\right), D^{\text {tang }} g_{0}(0, \xi)\right\rangle
\end{aligned}
$$


and for every $\xi \in \partial \Omega_{0}$,

$$
\begin{aligned}
& G(0, \xi, 0,0)=G_{u}(0, \xi, 0,0)=G_{p_{i}}(0, \xi, 0,0), \quad i=1, \ldots, N, \\
& G_{t}(0, \xi, 0,0)=\left\langle D^{\operatorname{tang}}\left(\frac{D_{t} g_{0}(0, \xi)}{\frac{\partial g_{0}}{\partial \nu}(0, \xi)-g_{1}(0, \xi)}\right), D^{\text {tang }} g_{0}(0, \xi)\right\rangle \\
& -D_{t} g_{0}(0, \xi)\left(\frac{\frac{\partial^{2} u_{0}}{\partial \nu^{2}}(\xi)-\frac{\partial g_{1}}{\partial \nu}(0, \xi)}{\frac{\partial g_{0}}{\partial \nu}(0, \xi)-g_{1}(0, \xi)}\right)+D_{t} g_{1}(0, \xi)
\end{aligned}
$$

Proof. Taking (2.21) into account, we rewrite the first addendum of (2.22) as

$$
\begin{aligned}
& \left\langle\left(I+{ }^{t} D \Phi\right)^{-1} \nu,\left(I+{ }^{t} D \Phi\right)^{-1} D\left(u_{0}+\left\langle D u_{0}, \Phi\right\rangle+w\right)\right\rangle \\
& =\frac{\partial u_{0}}{\partial \nu}+\frac{\partial}{\partial \nu}\left\langle D u_{0}, \Phi\right\rangle+\frac{\partial w}{\partial \nu}-\left\langle\left({ }^{t} D \Phi\right) \nu, D u_{0}\right\rangle \\
& \quad-\left\langle\nu,\left({ }^{t} D \Phi\right) D u_{0}\right\rangle+R(\xi, D w, \Phi, D \Phi),
\end{aligned}
$$

where $R(\xi, p, q, r)$ and its derivatives with respect to $p_{i}, q_{i}, r_{i j}, i, j=1, \ldots, N$, vanish at $(\xi, 0,0,0)$.

In the above sum we have

$$
\frac{\partial}{\partial \nu}\left\langle D u_{0}, \Phi\right\rangle=\frac{\partial^{2} u_{0}}{\partial \nu^{2}} s, \quad\left({ }^{t} D \Phi\right) \nu=D^{\text {tang }} s,\left\langle\nu,\left({ }^{t} D \Phi\right) D u_{0}\right\rangle=0,
$$

where the last equality follows from $(D \Phi) \nu=0$. Substituting $s=\mathcal{F}_{0}(t, \xi, w)$ and taking (2.12) into account, we get

$$
\begin{aligned}
&\left\langle\left(I+{ }^{t} D \Phi\right)^{-1} \nu,\left(I+{ }^{t} D \Phi\right)^{-1} D\left(u_{0}+\left\langle D u_{0}, \Phi\right\rangle+w\right)\right\rangle \\
&= \frac{\partial u_{0}}{\partial \nu}+\frac{\partial w}{\partial \nu}+\frac{\partial^{2} u_{0}}{\partial \nu^{2}}\left(\frac{w}{\frac{\partial g_{0}(t, \xi)}{\partial \nu}-g_{1}(0, \xi)}\right) \\
&-\left\langle D^{\operatorname{tang}}\left(\frac{w}{\frac{\partial g_{0}(t, \xi)}{\partial \nu}-g_{1}(0, \xi)}\right), D^{\operatorname{tang}} u_{0}\right\rangle+\frac{\partial^{2} u_{0}}{\partial \nu^{2}}\left(\frac{g_{0}(t, \xi)-g_{0}(0, \xi)}{\frac{\partial g_{0}(t, \xi)}{\partial \nu}-g_{1}(0, \xi)}\right) \\
&-\left\langle D^{\operatorname{tang}}\left(\frac{g_{0}(t, \xi)-g_{0}(0, \xi)}{\frac{\partial g_{0}(t, \xi)}{\partial \nu}-g_{1}(0, \xi)}\right), D^{\text {tang }} u_{0}\right\rangle+R_{1}(t, \xi, w, D w),
\end{aligned}
$$

where $R_{1}(t, \xi, u, p)$ vanishes at $(0, \xi, 0,0)$ as well as its derivatives with respect to $t, u, p_{i}, i=1, \ldots, N$.

On the other hand, since $\left\langle\left({ }^{t} D \Phi\right) \nu, \nu\right\rangle=0$, we have

$$
g_{1}(t, \xi+\Phi)\left|\left(I+{ }^{t} D \Phi\right)^{-1} \nu\right|=g_{1}(t, \xi)+\left\langle D g_{1}(t, \xi), s \nu(\xi)\right\rangle+R_{3}(t, \xi, \Phi, D \Phi),
$$

where $R_{3}(t, \xi, p, q)$ and its derivatives with respect to $t, p_{i}, q_{i j}, i, j=1, \ldots, N$, vanish at $(t, \xi, 0,0)$. Replacing $s$ and $\Phi$ and taking (2.12) into account again, we get

$$
\begin{aligned}
& g_{1}(t, \xi+\Phi)\left|\left(I+{ }^{t} D \Phi\right)^{-1} \nu\right| \\
& \quad=g_{1}(t, \xi)+\frac{\partial g_{1}(t, \xi)}{\partial \nu}\left(\frac{w+g_{0}(t, \xi)-g_{0}(0, \xi)}{\frac{\partial g_{0}(t, \xi)}{\partial \nu}-g_{1}(0, \xi)}\right)+R_{4}(t, \xi, w, D w),
\end{aligned}
$$

where $R_{4}(t, \xi, u, p)$ and its derivatives with respect to $\left(t, u, p_{i}\right)$ vanish at $(0, \xi, 0,0)$. Summing up, we get the statement. 
2.3. The problem for $w$. The final system for $w$ is therefore

$$
\left\{\begin{array}{l}
w_{t}=\mathcal{A}(t, \partial) w+F(t, w(t, \cdot)), \quad t \geq 0, \xi \in \bar{\Omega}_{0}, \\
\mathcal{B} w=G(t, w(t, \cdot)), \quad t \geq 0, \xi \in \partial \Omega_{0}, \\
w(0, \xi)=0, \quad \xi \in \bar{\Omega}_{0},
\end{array}\right.
$$

where we have set

$$
\begin{gathered}
F(t, v)(\xi)=\mathcal{F}(t, v)(\xi)-\mathcal{A}(t, \partial) v(\xi), \\
G(t, v)(\xi)=G(t, \xi, v(\xi), D v(\xi)), \quad \xi \in \partial \Omega_{0} .
\end{gathered}
$$

Problem (2.26) will be solved by a fixed point theorem in a ball of the parabolic Hölder space $C^{1+\alpha / 2,2+\alpha}\left([0, \delta] \times \bar{\Omega}_{0}\right)$, with $\delta$ small. The main tool is the next theorem about Hölder regularity and estimates for solutions of linear problems.

Theorem 2.3. Let $\alpha \in(0,1)$ and $\delta_{0}>0$. Let $\Omega$ be an open set in $\mathbb{R}^{N}$ with uniformly $C^{2+\alpha}$ boundary, and let $\alpha_{i j}, \beta_{i}, \gamma, \alpha_{i j}^{\prime}, \beta_{i}^{\prime}, \gamma^{\prime} \in C^{\alpha / 2, \alpha}\left(\left[0, \delta_{0}\right] \times \bar{\Omega}\right)$ be such that

$$
\sum_{i, j=1}^{N} \alpha_{i j}(t, \xi) \eta_{i} \eta_{j} \geq \nu|\eta|^{2}, \quad \xi \in \bar{\Omega}, 0 \leq t \leq \delta_{0}
$$

for some $\nu>0$, and

$$
\alpha_{i j}^{\prime}(0, \xi)=0, \quad \xi \in \bar{\Omega} .
$$

Moreover, let $\phi_{i}, \psi \in C^{1 / 2+\alpha / 2,1+\alpha}\left(\left[0, \delta_{0}\right] \times \partial \Omega\right)$ be such that

$$
\sum_{i=1}^{N} \phi_{i}(t, \xi) \nu_{i}(\xi) \neq 0, \quad \xi \in \partial \Omega, 0 \leq t \leq \delta_{0}
$$

For every $v \in C^{2}(\bar{\Omega})$ set

$$
\begin{aligned}
(\mathcal{A}(t, \partial) v)(\xi)= & \sum_{i, j=1}^{N}\left(\alpha_{i j}(t, \xi) D_{i j} v(\xi)+\alpha_{i j}^{\prime}(t, \xi) D_{i j} v\left(\xi^{\prime}\right)\right) \\
& +\sum_{i=1}^{N}\left(\beta_{i}(t, \xi) D_{i} v(\xi)+\beta_{i}^{\prime}(t, \xi) D_{i} v\left(\xi^{\prime}\right)\right) \\
& +\gamma(t, \xi) v(\xi)+\gamma^{\prime}(t, \xi) v\left(\xi^{\prime}\right), 0 \leq t \leq \delta_{0}, \xi \in \bar{\Omega}
\end{aligned}
$$

and

$$
(\mathcal{B}(t, \partial) v)(\xi)=\psi(t, \xi) v(\xi)+\sum_{i=1}^{N} \phi_{i}(t, \xi) D_{i} v(\xi), \quad 0 \leq t \leq \delta_{0}, \quad \xi \in \partial \Omega .
$$

Then there is $\delta_{1} \in\left(0, \delta_{0}\right]$ such that for every $f_{1} \in C^{\alpha / 2, \alpha}([0, \delta] \times \bar{\Omega})$ and every $g_{1} \in$ $C^{1 / 2+\alpha / 2,1+\alpha}([0, \delta] \times \partial \Omega)$ with $0<\delta \leq \delta_{1}$, satisfying the compatibility condition

$$
g_{1}(0, \cdot)=0 \quad \text { in } \partial \Omega
$$


the problem

$$
\left\{\begin{array}{l}
v_{t}=\mathcal{A}(t, \partial) v+f_{1}, \quad 0 \leq t \leq \delta, \xi \in \bar{\Omega} \\
\mathcal{B}(t, \partial) v=g_{1}, \quad t \geq 0, \xi \in \partial \Omega \\
v(0, x)=0, \quad \xi \in \bar{\Omega}
\end{array}\right.
$$

has a unique solution $v \in C^{1+\alpha / 2,2+\alpha}([0, \delta] \times \bar{\Omega})$. Moreover, there exists $C>0$, independent of $\delta$, such that

$$
\|v\|_{C^{1+\alpha / 2,2+\alpha}([0, \delta] \times \bar{\Omega})} \leq C\left(\left\|f_{1}\right\|_{C^{\alpha / 2, \alpha}([0, \delta] \times \bar{\Omega})}+\left\|g_{1}\right\|_{C^{1 / 2+\alpha / 2,1+\alpha}([0, \delta] \times \partial \Omega)}\right) .
$$

If in addition $\Omega$ has uniformly $C^{3+\alpha}$ boundary, and $\alpha_{i j}, \beta_{i}, \gamma, \alpha_{i j}^{\prime}, \beta_{i}^{\prime}, \gamma^{\prime}$ belong to $C^{1 / 2+\alpha / 2,1+\alpha}\left(\left[0, \delta_{0}\right] \times \bar{\Omega}\right), \phi_{i}, \psi \in C^{1+\alpha / 2,2+\alpha}\left(\left[0, \delta_{0}\right] \times \partial \Omega\right)$, then there is $\delta_{1} \leq \delta_{0}$ such that if $f_{1} \in C^{1 / 2+\alpha / 2,1+\alpha}([0, \delta] \times \bar{\Omega}), g_{1} \in C^{1+\alpha / 2,2+\alpha}([0, \delta] \times \partial \Omega)$ with $0<\delta \leq$ $\delta_{1}$ satisfy the compatibility condition (2.27) and the further compatibility condition

$$
\mathcal{B}(0, \partial) f_{1}(0, \cdot)=\frac{\partial g_{1}}{\partial t}(0, \cdot) \quad \text { in } \partial \Omega
$$

then the solution $v$ of problem (2.28) is in $C^{3 / 2+\alpha / 2,3+\alpha}([0, \delta] \times \bar{\Omega})$. Moreover, there exists $C>0$, independent of $\delta$, such that

$$
\|v\|_{C^{3 / 2+\alpha / 2,3+\alpha}([0, \delta] \times \bar{\Omega})} \leq C\left(\left\|f_{1}\right\|_{C^{1 / 2+\alpha / 2,1+\alpha}([0, \delta] \times \bar{\Omega})}+\left\|g_{1}\right\|_{C^{1+\alpha / 2,2+\alpha}([0, \delta] \times \partial \Omega)}\right) .
$$

Proof. If the coefficients $\alpha_{i j}^{\prime}, \beta_{i}^{\prime}, \gamma^{\prime}$ vanish, the result is well known and we can take $\delta_{1}=\delta_{0}$; see e.g. 111 for bounded $\Omega$, 17 for unbounded $\Omega$. In the general case the result follows by a (rather standard) perturbation argument, which we sketch below.

We write $\mathcal{A}=\mathcal{A}_{0}+\mathcal{A}_{1}$, where

$$
\begin{gathered}
\left(\mathcal{A}_{0}(t, \partial) v\right)(\xi)=\sum_{i, j=1}^{n} \alpha_{i j}(t, \xi) D_{i j} v(\xi)+\sum_{i=1}^{n} \beta_{i}(t, \xi) D_{i} v(\xi)+\gamma(t, \xi) v(\xi) \\
\left(\mathcal{A}_{1}(t, \partial) v\right)(\xi)=\sum_{i, j=1}^{n} \alpha_{i j}^{\prime}(t, \xi) D_{i j} v\left(\xi^{\prime}\right)+\sum_{i=1}^{n} \beta_{i}^{\prime}(t, \xi) D_{i} v\left(\xi^{\prime}\right)+\gamma^{\prime}(t, \xi) v\left(\xi^{\prime}\right)
\end{gathered}
$$

and we consider the operator $\mathcal{A}$ as a perturbation of $\mathcal{A}_{0}$. So, we look for a solution of (2.28) as a fixed point of the operator $\Lambda: X=\left\{u \in C^{1+\alpha / 2,2+\alpha}([0, \delta] \times \bar{\Omega})\right.$ : $u(0, \cdot)=0\} \mapsto X$, defined by $\Lambda u=v, v$ being the solution of

$$
\left\{\begin{array}{l}
v_{t}=\mathcal{A}_{0}(t, \partial) v+\mathcal{A}_{1}(t, \partial) u+f_{1}, \quad 0 \leq t \leq \delta, x \in \bar{\Omega} \\
\mathcal{B}(t, \partial) v=g_{1}, \quad t \geq 0, x \in \partial \Omega \\
v(0, x)=0, \quad x \in \bar{\Omega} .
\end{array}\right.
$$


We shall show that if $\delta$ is small enough, then $\Lambda$ is a $1 / 2$-contraction in $X$. If $u_{1}$, $u_{2} \in X$, then $\Lambda u_{1}-\Lambda u_{2}$ is the solution $v$ of

$$
\left\{\begin{array}{l}
v_{t}=\mathcal{A}_{0}(t, \partial) v+\mathcal{A}_{1}(t, \partial)\left(u_{1}-u_{2}\right), \quad 0 \leq t \leq \delta, x \in \bar{\Omega}, \\
\mathcal{B}(t, \partial) v=0, \quad t \geq 0, x \in \partial \Omega, \\
v(0, x)=0, \quad x \in \bar{\Omega},
\end{array}\right.
$$

so that $\left\|\Lambda u_{1}-\Lambda u_{2}\right\|_{X} \leq C\left\|\mathcal{A}_{1}(t, \partial)\left(u_{1}-u_{2}\right)\right\|_{C^{\alpha / 2, \alpha}([0, \delta] \times \bar{\Omega})}$. It is easy to see that there is $C>0$ such that

$$
\left\|\sum_{i=1}^{n} \beta_{i}^{\prime} D_{i}\left(u_{1}-u_{2}\right)+\gamma^{\prime}\left(u_{1}-u_{2}\right)\right\| \leq C \delta^{\alpha / 2}\left\|u_{1}-u_{2}\right\|_{C^{1+\alpha / 2,2+\alpha}([0, \delta] \times \bar{\Omega})} .
$$

Moreover, since $\alpha_{i j}^{\prime}(0, \cdot)=0$ and $D_{i j}\left(u_{1}-u_{2}\right)(0, \cdot)=0$, we have $\left\|\alpha_{i j}^{\prime}\right\|_{\infty} \leq$ $\delta^{\alpha / 2}\left[\alpha_{i j}^{\prime}\right]_{C^{\alpha / 2, \alpha}},\left\|D_{i j}\left(u_{1}-u_{2}\right)\right\|_{\infty} \leq \delta^{\alpha / 2}\left[u_{1}-u_{2}\right]_{C^{1+\alpha / 2,2+\alpha}}$. Therefore,

$$
\left\|\alpha_{i j}^{\prime} D_{i j}\left(u_{1}-u_{2}\right)\right\|_{\infty} \leq C \delta^{\alpha / 2}\left\|u_{1}-u_{2}\right\|_{C^{1+\alpha / 2,2+\alpha}},
$$

and

$$
\begin{aligned}
& {\left[\alpha_{i j}^{\prime} D_{i j}\left(u_{1}-u_{2}\right)\right]_{C^{\alpha / 2, \alpha}}} \\
& \quad \leq\left\|\alpha_{i j}^{\prime}\right\|_{\infty}\left[D_{i j}\left(u_{1}-u_{2}\right)\right]_{C^{\alpha / 2, \alpha}}+\left[\alpha_{i j}^{\prime}\right]_{C^{\alpha / 2, \alpha}}\left\|D_{i j}\left(u_{1}-u_{2}\right)\right\|_{\infty} \\
& \quad \leq C \delta^{\alpha / 2}\left\|u_{1}-u_{2}\right\|_{C^{1+\alpha / 2,2+\alpha}([0, \delta] \times \bar{\Omega})} .
\end{aligned}
$$

Therefore, for $\delta$ small enough, $\Lambda$ is a $1 / 2$-contraction, and the first part of the statement follows. The proof of the second part is similar.

Now we are ready to solve problem (2.26).

Theorem 2.4. There is $R_{0}>0$ such that for every $R \geq R_{0}$ and for every sufficiently small $\delta>0$ problem (2.26) has a unique solution in the ball $B(0, R) \subset$ $C^{1+\alpha / 2,2+\alpha}\left([0, \delta] \times \bar{\Omega}_{0}\right)$.

Proof. Let $\delta_{0}, r>0$ be such that $\mathcal{F}(t, v)$ is defined for $t \in\left[0, \delta_{0}\right]$ and for $v \in C^{2}\left(\bar{\Omega}_{0}\right)$ with $\|v\|_{C^{1}} \leq r$. Fix $R>0$ and define $Y$ to be the space

$$
Y=\left\{v \in C^{1+\alpha / 2,2+\alpha}\left([0, \delta] \times \bar{\Omega}_{0}\right), v(0, \cdot)=0,\|v\|_{C^{1+\alpha / 2,2+\alpha}} \leq R\right\},
$$

where $\delta \in\left(0, \delta_{0}\right]$ is so small that $\|v\|_{C^{1+\alpha / 2,2+\alpha}} \leq R$ implies $\|v(t, \cdot)\|_{C^{1}} \leq r$ for every $t \in[0, \delta]$. Therefore $F(t, v(t, \cdot))$ is well defined for all $t \in[0, \delta]$ and $v \in Y$.

For any fixed $v \in Y$, we introduce the linear problem

$$
\left\{\begin{array}{l}
w_{t}=\mathcal{A}(t, \partial) w+F(t, v(t, \cdot)), \quad 0 \leq t \leq \delta, x \in \bar{\Omega}_{0}, \\
\mathcal{B} w=G(t, v(t, \cdot)), \quad 0 \leq t \leq \delta, x \in \partial \Omega_{0}, \\
w(0, x)=0, \quad x \in \bar{\Omega}_{0},
\end{array}\right.
$$

to which we apply Theorem 2.3 Since the compatibility condition $G(0, v(0))=0$ is satisfied, for $\delta \leq \delta_{1}$ (2.32) has a unique solution $w \in C^{1+\alpha / 2,2+\alpha}\left([0, \delta] \times \bar{\Omega}_{0}\right)$.

We define $\Gamma(v)=w, w$ being the solution of (2.32). Our aim is to find a local solution to (2.26) as a fixed point of $\Gamma$.

We shall prove that for suitable $\delta$ and $R$ (roughly, $R$ large and $\delta$ small), $\Gamma$ maps $Y$ into itself and is a contraction. 
For $u, v \in Y$ the function $w=\Gamma(v)-\Gamma(u)$ is the solution of

$$
\left\{\begin{array}{l}
w_{t}=\mathcal{A} w+F(t, v(t, \cdot))-F(t, u(t, \cdot)), \quad 0 \leq t \leq \delta, \xi \in \bar{\Omega}_{0}, \\
\mathcal{B} w=G(t, v(t, \cdot))-G(t, u(t, \cdot)), \quad 0 \leq t \leq \delta, \xi \in \partial \Omega_{0}, \\
w(0, \xi)=0, \quad \xi \in \bar{\Omega}_{0} .
\end{array}\right.
$$

We have therefore the estimate

$$
\|w\|_{C^{1+\alpha / 2,2+\alpha}} \leq C\left(\|F(t, v)-F(t, u)\|_{C^{\alpha / 2, \alpha}}+\|G(t, v)-G(t, u)\|_{C^{1 / 2+\alpha / 2,1+\alpha}}\right)
$$

with $C$ independent of $\delta$. We claim now that

$$
\begin{aligned}
& \|F(t, v)-F(t, u)\|_{C^{\alpha / 2, \alpha}}+\|G(t, v)-G(t, u)\|_{C^{1 / 2+\alpha / 2,1+\alpha}} \\
& \quad \leq K(R) \delta^{\alpha / 2}\|u-v\|_{C^{1+\alpha / 2,2+\alpha}},
\end{aligned}
$$

where $K$ is an increasing function. The proof of (2.33) is straightforward although lengthy; it may be found in [14, Ch. 8] in the case of a local $F$.

Once (2.33) is established, we get immediately that $\Gamma$ is a $1 / 2$-contraction provided

$$
C K(R) \delta^{\alpha / 2} \leq \frac{1}{2}
$$

In this case, for each $u \in Y$ we have

$$
\|\Gamma(u)\|_{C^{1+\alpha / 2,2+\alpha}} \leq\|\Gamma(0)\|_{C^{1+\alpha / 2,2+\alpha}}+\frac{R}{2},
$$

where $\Gamma(0)$ is the solution of

$$
\left\{\begin{array}{l}
w_{t}=\mathcal{A}(t, \partial) w+F(t, 0), \quad 0 \leq t \leq \delta, x \in \bar{\Omega}_{0}, \\
\mathcal{B} w=G(t, 0), \quad 0 \leq t \leq \delta, x \in \partial \Omega_{0} \\
w(0, x)=0, \quad x \in \bar{\Omega}_{0}
\end{array}\right.
$$

so that

$$
\|\Gamma(0)\|_{C^{1+\alpha / 2,2+\alpha}} \leq C\left(\|F(t, 0)\|_{C^{\alpha / 2, \alpha}}+\|G(t, 0)\|_{C^{1 / 2+\alpha / 2,1+\alpha}}\right) .
$$

If $R$ is so large that

$$
C\left(\|F(t, 0)\|_{C^{\alpha / 2, \alpha}\left(\left[0, \delta_{0}\right] \times \bar{\Omega}_{0}\right)}+\|G(t, 0)\|_{C^{1 / 2+\alpha / 2,1+\alpha}\left(\left[0, \delta_{0}\right] \times \partial \Omega_{0}\right)}\right) \leq \frac{R}{2},
$$

then for every $u \in Y$ we have $\|\Gamma(u)\|_{C^{1+\alpha / 2,2+\alpha}} \leq R$, and $\Gamma$ maps $Y$ into itself. Therefore $\Gamma$ has a unique fixed point in $Y$.

Since the problem for $w$ is parabolic, it is natural to have further regularity results, depending on the regularity of the data. Here we prove two further regularity theorems: a time-space regularity theorem up to $t=0$, which will be used later, and a smoothness theorem for $t>0$.

The Hölder time-space regularity theorem needs the following regularity and compatibility assumptions:

(d) Further regularity assumptions. $\Omega_{0} \subset \mathbb{R}^{N}$ is a nonempty open set with uniformly $C^{4+\alpha}$ boundary $\partial \Omega_{0}$, and $u_{0} \in C^{4+\alpha}\left(\bar{\Omega}_{0}\right)$. The coefficients $a_{i j}$ and their first order space derivatives belong to $C^{1 / 2+\alpha / 2,1+\alpha}\left([0, T] \times \mathbb{R}^{N}\right)$. The function $f=f(t, x, u, p)$ is differentiable with respect to $x$, twice differentiable with respect 
to $(u, p) ; f$ and its derivatives are continuous in $(t, x, u, p)$, and $C^{1 / 2+\alpha / 2,1+\alpha}$ in $(t, x)$, locally uniformly with respect to $(u, p)$. Moreover, $g_{0}$ and $g_{1}$ are differentiable with respect to the space variables, and $g_{0}, D_{i} g_{0} \in C^{3 / 2+\alpha / 2,3+\alpha}\left([0, T] \times \mathbb{R}^{N}\right)$, $g_{1}, D_{i} g_{1} \in C^{1+\alpha / 2,2+\alpha}\left([0, T] \times \mathbb{R}^{N}\right)$ for $i=1, \ldots, N$.

(e) Further compatibility conditions. Let $\mathcal{B}$ be the operator defined in (2.24). We assume that for every $\xi \in \partial \Omega_{0}$

$$
\mathcal{B}\left(\mathcal{L}(0, \partial) u_{0}+f\left(0, \cdot, u_{0}, D u_{0}\right)\right)=\mathcal{B} \frac{\partial g_{0}}{\partial t}(0, \cdot)-\frac{\partial}{\partial \nu}\left(\frac{\partial g_{0}}{\partial t}(0, \cdot)\right)+\frac{\partial g_{1}}{\partial t}(0, \cdot)
$$

This condition comes out from $\frac{\partial u}{\partial n}-g_{1}=0$ on $\partial \Omega_{t}$, differentiating with respect to time and taking the trace on $\partial \Omega_{0}$ at $t=0$. It is necessary that $\frac{\partial}{\partial t} \frac{\partial u}{\partial n}$ be continuous up to $t=0$.

Theorem 2.5. Let assumptions (a), (b), (c), (d), (e) hold, and let $w:[0, \delta] \times$ $\bar{\Omega}_{0} \rightarrow \mathbb{R}$ be the solution to problem (2.26) given by Theorem 2.4. Then there exists $\delta^{\prime} \in(0, \delta)$ such that $w$ belongs to $C^{1 / 2+\alpha / 2,3+\alpha}\left(\left[0, \delta^{\prime}\right] \times \bar{\Omega}_{0}\right)$.

Sketch of the proof. Let us go back to the proof of Theorem 2.4. Now we look for a fixed point of $\Gamma$ in the space

$$
Y^{\prime}=\left\{v \in C^{3 / 2+\alpha / 2,3+\alpha}\left([0, \delta] \times \bar{\Omega}_{0}\right), v(0, \cdot)=0,\|v(t, \cdot)\|_{C^{3 / 2+\alpha / 2,3+\alpha}} \leq R^{\prime}\right\}
$$

which is closed in $C^{1+\alpha / 2,2+\alpha}\left([0, \delta] \times \bar{\Omega}_{0}\right)$. If we prove that $\Gamma$ maps $Y^{\prime}$ into itself for $R^{\prime}$ large and $\delta$ small, we are done, because from the proof of Theorem 2.4 we already know that $\Gamma$ is a $1 / 2$-contraction with respect to the $C^{1+\alpha / 2,2+\alpha}$ distance. To this aim we use the last part of Theorem 2.3 It yields that

$$
\Gamma v \in C^{3 / 2+\alpha / 2,3+\alpha}\left([0, \delta] \times \bar{\Omega}_{0}\right)
$$

provided

$$
\begin{aligned}
& \varphi(t, \xi)=F(t, v(t, \cdot))(\xi) \in C^{1 / 2+\alpha / 2,1+\alpha}\left([0, \delta] \times \bar{\Omega}_{0}\right), \\
& \psi(t, \xi)=G(t, v(t, \cdot))(\xi) \in C^{1+\alpha / 2,2+\alpha}\left([0, \delta] \times \partial \Omega_{0}\right),
\end{aligned}
$$

and that the compatibility condition

$$
\mathcal{B} F(0,0)=\frac{d}{d t} G(t, v(t))_{\mid t=0}
$$

holds. In this case, it gives also the estimate

$\|\Gamma v\|_{C^{3 / 2+\alpha / 2,3+\alpha}\left([0, \delta] \times \bar{\Omega}_{0}\right)} \leq C\left(\|\varphi\|_{C^{1 / 2+\alpha / 2,1+\alpha}\left([0, \delta] \times \bar{\Omega}_{0}\right)}+\|\psi\|_{C^{1+\alpha / 2,2+\alpha}\left([0, \delta] \times \partial \Omega_{0}\right)}\right)$.

The compatibility condition (2.34) holds thanks to assumption (e). From the regularity assumptions $(\mathrm{d})$ we get easily that $\varphi \in C^{1 / 2+\alpha / 2,1+\alpha}\left([0, \delta] \times \bar{\Omega}_{0}\right)$, and that $\psi \in C^{1+\alpha / 2,2+\alpha}\left([0, \delta] \times \partial \Omega_{0}\right)$. A lengthy but elementary estimation shows that

$$
\begin{aligned}
\|(t, \xi) & \mapsto \varphi(t, \xi)-\varphi(0, \xi)\left\|_{C^{1 / 2+\alpha / 2,1+\alpha}\left([0, \delta] \times \bar{\Omega}_{0}\right)} \leq C_{1} \delta^{\alpha / 2}\right\| v \|_{C^{3 / 2+\alpha / 2,3+\alpha}\left([0, \delta] \times \bar{\Omega}_{0}\right)}, \\
\|(t, \xi) & \mapsto \psi(t, \xi)-\psi(0, \xi)\left\|_{C^{1+\alpha / 2,2+\alpha}\left([0, \delta] \times \partial \Omega_{0}\right)} \leq C_{2} \delta^{\alpha / 2}\right\| v \|_{C^{3 / 2+\alpha / 2,3+\alpha}\left([0, \delta] \times \bar{\Omega}_{0}\right)},
\end{aligned}
$$

for every $v \in Y^{\prime}$. It follows that

$$
\begin{aligned}
& \quad\|\Gamma v\|_{C^{3 / 2+\alpha / 2,3+\alpha}\left([0, \delta] \times \bar{\Omega}_{0}\right)} \\
& \quad \leq C\left(\|F(t, 0)\|_{C^{1 / 2+\alpha / 2,1+\alpha}\left([0, \delta] \times \bar{\Omega}_{0}\right)}\right. \\
& \left.\quad+\|G(t, 0)\|_{C^{1+\alpha / 2,2+\alpha}\left([0, \delta] \times \partial \Omega_{0}\right)}+\left(C_{1}+C_{2}\right) \delta^{\alpha / 2} R^{\prime}\right),
\end{aligned}
$$

so that $\Gamma$ maps $Y^{\prime}$ into itself if $R^{\prime}$ is large enough and $\delta$ is small enough. 
Now we prove local time smoothness of $w$. Of course we need some smoothness assumptions on the data. Together with assumptions (a), (b), (c), we shall consider

(f) Smoothness assumptions. The coefficients $a_{i j}, b_{i}, c$, and the functions $f, g_{0}$, $g_{1}$ are $C^{\infty}$ for $t>0$.

Theorem 2.6. Let (a), (b), (c), (f) hold, and let $w:[0, \delta] \times \bar{\Omega}_{0} \rightarrow \mathbb{R}$ be the solution to problem (2.26) given by Theorem 2.4. Then there exists $\delta^{\prime} \in(0, \delta)$ such that the function $t \mapsto w(t, \cdot)$ is in $C^{\infty}\left(\left(0, \delta^{\prime}\right) ; C^{2}\left(\bar{\Omega}_{0}\right)\right)$.

Proof. Let $\delta^{\prime} \in(0, \delta)$. For every $\eta$ sufficiently close to 1 the function $w_{\eta}(t, \cdot)=$ $w(t \eta, \cdot)$ is well defined for $t \in[0, \delta]$, and it is a solution of

$$
\Psi(\eta, v)(t)=\left(v_{t}-\eta \mathcal{A}(t, \partial) v-\eta F(t, v(t, \cdot)) ; \mathcal{B} v-G(t, v(t, \cdot))\right)=(0,0) .
$$

$\Psi$ may be seen as a function from $[1-r, 1+r] \times\left\{v \in C^{1+\beta / 2,2+\beta}\left(\left[0, \delta^{\prime}\right] \times \bar{\Omega}_{0}\right)\right.$ : $v(0, \cdot)=0,\|v\| \leq \rho\}$ to $C^{\beta / 2, \beta}\left(\left[0, \delta^{\prime}\right] \times \bar{\Omega}\right) \times C^{1 / 2+\beta / 2,1+\beta}\left(\left[0, \delta^{\prime}\right] \times \partial \Omega_{0}\right)$, for every $\beta \in(0, \alpha]$, and for every small $\rho>0$.

At $(\eta, v)=(1, w)$ we have of course $\Psi(1, w)=(0,0) . \Psi$ is smooth, and at $(1, w)$ the derivative of $\Psi$ with respect to $v$ is given by

$$
\left(\Psi_{v}(1, w) v\right)(t)=\left(v_{t}-\mathcal{A}(t, \partial) v-F_{v}(t, w(t, \cdot)) v ; \mathcal{B} v-G_{v}(t, w(t, \cdot)) v\right) .
$$

By Theorem [2.3, it is an isomorphism between $\left\{v \in C^{1+\beta / 2,2+\beta}\left(\left[0, \delta^{\prime}\right] \times \bar{\Omega}_{0}\right)\right.$ : $v(0, \cdot)=0\}$ and $C^{\beta / 2, \beta}\left(\left[0, \delta^{\prime}\right] \times \bar{\Omega}_{0}\right) \times C^{1 / 2+\beta / 2,1+\beta}\left(\left[0, \delta^{\prime}\right] \times \partial \Omega_{0}\right)$, provided $\delta^{\prime}$ is small enough.

Therefore by the implicit function theorem there are $r_{1}, r_{2}>0$ and a smooth function

$$
\psi:\left(1-r_{1}, 1+r_{1}\right) \mapsto B\left(w, r_{2}\right) \subset\left\{v \in C^{1+\beta / 2,2+\beta}\left(\left[0, \delta^{\prime}\right] \times \bar{\Omega}_{0}\right): v(0, \cdot)=0\right\}
$$

such that for $\eta \in\left(1-r_{1}, 1+r_{1}\right)$ and $v \in B\left(w, r_{2}\right)$ with $\Psi(\eta, v)=(0,0)$ we have $v=\psi(\eta)$.

Fix $\beta \in(0, \alpha)$. Then we have

$$
\lim _{\eta \rightarrow 1} w_{\eta}=w \quad \text { in } C^{1+\beta / 2,2+\beta}\left(\left[0, \delta^{\prime}\right] \times \bar{\Omega}_{0}\right),
$$

so that $w_{\eta}$ belongs to $B\left(w, r_{2}\right) \subset C^{1+\beta / 2,2+\beta}\left(\left[0, \delta^{\prime}\right] \times \bar{\Omega}_{0}\right)$ if $\eta$ is sufficiently close to 1 . Since $\Psi\left(\eta, w_{\eta}\right)=(0,0)$, then $w_{\eta}=\psi(\eta)$. It follows that for $\eta$ close to 1 , the function $\eta \mapsto w(t \eta, \xi)$ is smooth with values in $C^{1,2}\left(\left[0, \delta^{\prime}\right] \times \bar{\Omega}_{0}\right)$. This implies easily that $w$ and its first and second order space derivatives are smooth with respect to time for $0<t<\delta^{\prime}$.

The idea of introducing the parameter $\eta$ to prove time regularity of the solution is not new; see [2].

Remark 2.7. Throughout the section we have considered only problems with initial time $t_{0}=0$, but it is clear that the results of Theorems 2.4, 2.5] and 2.6 still hold for the problem

$$
\left\{\begin{array}{l}
w_{t}=\mathcal{A}(t, \partial) w+F_{0}(t, w(t, \cdot)), \quad t \geq t_{0}, \xi \in \bar{\Omega}_{0} \\
\mathcal{B}_{0} w=G_{0}(t, w(t, \cdot)), \quad t \geq t_{0}, \xi \in \partial \Omega_{0} \\
w\left(t_{0}, \xi\right)=0, \quad \xi \in \bar{\Omega}_{0}
\end{array}\right.
$$


with initial time $t_{0} \in(0, T)$, provided the due compatibility and transversality conditions are satisfied at $t=t_{0}$. Here $\mathcal{B}_{0}, F_{0}$ and $G_{0}$ are defined as $\mathcal{B}, F$ and $G$, with $g_{0}(0, \xi)$ and $g_{1}(0, \xi)$ replaced by $g_{0}\left(t_{0}, \xi\right)$ and $g_{1}\left(t_{0}, \xi\right)$.

Once we have locally solved (2.26) we come back to the original problem (1.1), using (2.11) and (2.1) to define $\partial \Omega_{t}$. Note that $s$ has the same regularity as $w$, i.e. it is in $C^{1+\alpha / 2,2+\alpha}\left([0, \delta] \times \partial \Omega_{0}\right)$ under assumptions (a), (b), (c); it is in $C^{3 / 2+\alpha / 2,3+\alpha}\left([0, \delta] \times \partial \Omega_{0}\right)$ under assumptions (a), (b), (c), (d), (e); it is smooth with respect to time for $t>0$ under assumptions (a), (b), (c), (f). Then we define $\widetilde{u}$ through (2.7), where $\Phi$ is given by (2.3). Again, $\widetilde{u}$ has the same regularity as $w$. As a last step we define $u$ through the change of coordinates, $u(t, x)=\widetilde{u}(t, \xi)$, where $x=\xi+\Phi(t, \xi)$. We have thus proved the following existence and regularity result.

Theorem 2.8. Let (a), (b), (c) hold. Then there is $\delta>0$ such that problem (1.1) has a solution $\left(\Omega_{t}, u\right)$ such that the $N+1$-dimensional hypersurface $\mathcal{S}=\{(t, x)$ : $\left.0 \leq t \leq \delta, x \in \partial \Omega_{t}\right\}$ and each $\partial \Omega_{t}$ are of class $C^{1+\alpha / 2,2+\alpha}$, and the function $u:\left\{(t, x) ; 0 \leq t \leq \delta, x \in \bar{\Omega}_{t}\right\} \mapsto \mathbb{R}$ is of class $C^{1+\alpha / 2,2+\alpha}$.

If also $(d),(e)$ hold, then $\mathcal{S}$ and each $\partial \Omega_{t}$ are of class $C^{3 / 2+\alpha / 2,3+\alpha}$, and the function $u$ is of class $C^{3 / 2+\alpha / 2,3+\alpha}$.

If (a), (b), (c), (f) hold, then the free boundary is $C^{\infty}$ with respect to time for $t>0$, as well as $u$ and its first and second order space derivatives.

\section{UNIQUENESS AND TIME SMOOTHNESS}

We say that a family of open sets $\left\{\Omega_{t}: a \leq t \leq b\right\} \subset \mathbb{R}^{N}$ is of class $C^{1+\alpha / 2,2+\alpha}$ in the interval $[a, b]$ if each boundary $\partial \Omega_{t}$ and the $(N+1)$-dimensional hypersurface $\left\{(t, x): a \leq t \leq b, x \in \partial \Omega_{t}\right\}$ are of class $C^{1+\alpha / 2,2+\alpha}$.

As a consequence of the uniqueness part of Theorem 2.4 we get uniqueness of regular solutions to problems of the type (1.1).

Corollary 3.1. Let assumptions (a), (b), (c) hold, and let $\left(\Omega_{t}^{(1)}, u^{(1)}(t, x)\right)$ and $\left(\Omega_{t}^{(2)}, u^{(2)}(t, x)\right)$ be two solutions to (1.1) of class $C^{1+\alpha / 2,2+\alpha}$ in an interval $[0, a]$, with space derivatives of class $\left.C^{1+\alpha / 2,2+\alpha} 1\right]$ Assume in addition that $\partial g_{0} / \partial n(t, x) \neq$ $g_{1}(t, x)$ for every $t \in[0, a], x \in \partial \Omega_{t}^{(1)}$, where $n(t, x)$ is the exterior unit normal vector to $\partial \Omega_{t}^{(1)}$ at the point $x$. Then $\left(\Omega_{t}^{(1)}, u^{(1)}(t, \cdot)\right)=\left(\Omega_{t}^{(2)}, u^{(2)}(t, \cdot)\right)$ for every $t \in[0, a]$.

Let assumption $(f)$ hold in addition, and let $\left(\Omega_{t}, u(t, x)\right)$ be any solution to (1.1) of class $C^{1+\alpha / 2,2+\alpha}$ in an interval $[0, a]$, with space derivatives of class $C^{1+\alpha / 2,2+\alpha}$, and such that $\partial g_{0} / \partial n(t, x) \neq g_{1}(t, x)$ for every $t \in[0, a]$. Then $\partial \Omega_{t}$ and $u$ are $C^{\infty}$ with respect to $t$ in $(0, a)$.

Proof. Let us prove uniqueness. Let $t_{0}$ be the supremum of the numbers $t \in[0, a]$ such that $\Omega_{s}^{(1)}=\Omega_{s}^{(2)}$ for $0 \leq s \leq t$ and $u^{(1)}(s, x)=u^{(2)}(s, x)$ for $0 \leq s \leq t$, $x \in \Omega_{s}^{(1)}=\Omega_{s}^{(1)}$. Then we have also $\Omega_{t_{0}}^{(1)}=\Omega_{t_{0}}^{(2)}$ and $u^{(1)}\left(t_{0}, x\right)=u^{(2)}\left(t_{0}, x\right)$.

\footnotetext{
${ }^{1}$ Thanks to Theorem 2.8 at least one solution with such regularity properties exists in a small interval if also assumptions (d), (e) are satisfied.
} 
Assume for a contradiction that $t_{0}<a$. Consider the initial value problem

$$
\left\{\begin{array}{l}
u_{t}(t, x)=\mathcal{L} u(t, x)+f(t, x), \quad t \geq t_{0}, x \in \Omega_{t}, \\
u(t, x)=g_{0}(t, x), \quad t \geq t_{0}, x \in \partial \Omega_{t}, \\
\frac{\partial u}{\partial n}(t, x)=g_{1}(t, x), \quad t \geq t_{0}, x \in \partial \Omega_{t}, \\
u\left(t_{0}, x\right)=u^{(1)}\left(t_{0}, x\right), \quad x \in \Omega_{t_{0}}^{(1)},
\end{array}\right.
$$

which can obviously be written as a problem with initial time 0 , with data satisfying all the assumptions (a), (b), (c) at the beginning of section 2. Following the construction of section 2, we arrive at two solutions $w^{(1)}, w^{(2)}$ of (2.35) in the interval $\left[t_{0}, a\right]$, belonging to $C^{1+\alpha / 2,2+\alpha}\left(\left[t_{0}, a\right] \times \bar{\Omega}_{t_{0}}^{(1)}\right)$ and with the property that for each $\delta \in\left(0, a-t_{0}\right]$ the restrictions of $w^{(1)}$ and $w^{(2)}$ to $\left[t_{0}, t_{0}+\delta\right] \times \bar{\Omega}_{t_{0}}^{(1)}$ do not coincide. Let $R=\max \left\{R_{0},\left\|w^{(1)}\right\|_{C^{1+\alpha / 2,2+\alpha}\left(\left[t_{0}, a\right] \times \bar{\Omega}_{t_{0}}^{(1)}\right)},\left\|w^{(2)}\right\|_{C^{1+\alpha / 2,2+\alpha}\left(\left[t_{0}, a\right] \times \bar{\Omega}_{t_{0}}^{(1)}\right)}\right\}$, where $R_{0}$ is given by Theorem 2.4 From Theorem 2.4 we know that if $\delta$ is sufficiently small, then (2.26) has a unique solution in the ball $B(0, R) \subset C^{1+\alpha / 2,2+\alpha}\left(\left[t_{0}, t_{0}+\delta\right] \times \bar{\Omega}_{t_{0}}^{(1)}\right)$, and this is a contradiction.

Let us prove time smoothness. By Theorem [2.6, for every $\bar{t} \in[0, a]$ there are $r=r(\bar{t})>0$ and $\delta=\delta(\bar{t})>0$ such the solution to problem (2.35) is in the space $C^{\infty}\left(\left(t_{0}, t_{0}+\delta\right) ; C^{2}\left(\bar{\Omega}_{0}\right)\right)$ for any $t_{0} \in[0, a] \cap[\bar{t}-r, \bar{t}+r]$. Fix any $\varepsilon \in(0, a)$. The union of the intervals $(\bar{t}, \bar{t}+\delta(\bar{t})) \cap[0, a]$ contains $[\varepsilon, a]$, and there are $\bar{t}_{1}, \ldots, \bar{t}_{n}$ such that the union $\bigcup_{i=1}^{n}\left(\bar{t}_{i}, \bar{t}_{i}+\delta\left(\bar{t}_{i}\right)\right) \cap[0, a]$ still contains $[\varepsilon, a]$. In each of these intervals, time smoothness of $w$ implies time smoothness of $s$ (hence, time smoothness of $\left.\partial \Omega_{t}\right)$ and time smoothness of $u$ and of its first and second order space derivatives. The statement follows.

Remark 3.2. Things may be considerably simplified if $\partial \Omega_{0}$ is the graph of a regular function, say $\Omega_{0}=\left\{\xi \in \mathbb{R}^{N}: \xi_{N} \leq \phi\left(\xi_{1}, \ldots, \xi_{n-1}\right)\right\}$ with $\phi \in C^{3+\alpha}\left(\mathbb{R}^{N-1}\right)$. In this case we may look for $\Omega_{t}$ of the form $\Omega_{t}=\left\{\xi \in \mathbb{R}^{N}: \xi_{N} \leq \phi\left(\xi_{1}, \ldots, \xi_{n-1}\right)+\right.$ $\left.s\left(t, \xi_{1}, \ldots, \xi_{n-1}\right)\right\}$, where the unknown $s$ is not in general the signed distance from $\partial \Omega_{0}$, but it works as well. The change of coordinates which transforms $\Omega_{t}$ into $\Omega_{0}$ is now trivial,

$$
\xi=\left(x_{1}, \ldots, x_{n-1}, x_{N}-s\left(t, x_{1}, \ldots, x_{n-1}\right)\right), \quad x \in \Omega_{t} .
$$

In addition to some simplifications in notation and formulas, the main advantage of this change of coordinates is that the procedure of Theorem 2.6 also gives time analyticity (in the case of analytic data, of course) for $t>0$. See e.g. Lorenzi [13, who used this method to get time analyticity in a system arising as a model in Combustion Theory, for initial data close to a travelling wave solution. Note that the map $\boldsymbol{\Psi}$ used in Theorem 2.6 is smooth but not analytic in general, because of the change of coordinates (2.4) which contains the cutoff function $\theta$ introduced in $\S 2.1$.

\section{ACKNOWLEDGEMENT}

We thank one of the referees for pointing out a mistake in the first version of the manuscript. 


\section{REFERENCES}

1. D. Andreucci, R. Gianni: Classical solutions to a multidimensional free boundary problem arising in combustion theory, Comm. Partial Differential Equations 19 (1994), 803-826. MR 95h:35248

2. S. Angenent: Analyticity of the interface of the porous media equation after the waiting time, Proc. Amer. Math. Soc. 102 (1988), 329-336. MR 89f:35103

3. C. M. Brauner, J. Hulshof, A. Lunardi: A general approach to stability in free boundary problems, J. Differential Equations 164 (2000), 16-48. MR 2001f:35438

4. C. M. Brauner, A. Lunardi: Instabilities in a combustion model with free boundary in $\mathbb{R}^{2}$, Arch. Rational Mech. Anal. 154 (2000), 157-182. MR 2001i:35293

5. C. M. Brauner, J. Hulshof, A. Lunardi: A critical case of stability in a free boundary problem, J. Evol. Eqns. 1 (2001), 85-113. MR 2002f:35228

6. J. D. Buckmaster, G. S. S. Ludford: Theory of Laminar Flames, Cambridge University Press, Cambridge, 1982. MR 84f:80011

7. L. A. Caffarelli, J. L. Vazquez: A free boundary problem for the heat equation arising in flame propagation, Trans. Amer. Math. Soc. 347 (1995), 411-441. MR 95e:35097

8. J. Escher, G. Simonett: Maximal regularity for a free boundary problem, Nonlinear Differential Equations Appl. 2 (1995), 463-510. MR 96k:35142

9. J. Escher, G. Simonett: Classical solutions of multidimensional Hele-Shaw models, SIAM J. Math. Anal. 28 (1997), 1028-1047. MR 98i:35213

10. V. A. Galaktionov, J. Hulshof, J. L. Vazquez: Extinction and focusing behaviour of spherical and annular flames described by a free boundary problem, J. Math. Pures Appl. 76 (1997), 563-608. MR 98h:35238

11. O. A. Ladyzhenskaja, V. A. Solonnikov, N. N. Ural'Ceva: Linear and quasilinear equations of parabolic type, Nauka, Moskow 1967 (Russian). English transl.: Transl. Math. Monographs, Amer. Math. Soc., Providence, RI, 1968. MR 39:3159b

12. C. Lederman, J. L. Vázquez, N. Wolanski: Uniqueness of solution to a free boundary problem from combustion, Trans. Amer. Math. Soc. 353 (2001), 655-692. MR 2001m:35327

13. L. LoRENZI: Regularity and analyticity in a two-dimensional combustion model, Adv. in Differential Equations 7 (2002), 1343-1376.

14. A. LunARDi: Analytic semigroup and optimal regularity in parabolic problems, BirkhäuserVerlag, Basel, 1995. MR 96e:47039

15. A. M. MeIRmanov: On a problem with free boundary for parabolic equations, Math. USSR Sbornik 43 (1982), 473-484. MR 83a:35059

16. M. Primicerio: Diffusion problems with a free boundary, Boll. Un. Mat. Ital. A (5) 18 (1981), 11-68. MR 82i:35166

17. V. A. SOLONNIKOV: On the boundary value problems for linear parabolic systems of differential equations of general form, Proc. Steklov Inst. Math. 83 (1965). English transl.: Boundary Value Problems of Mathematical Physics III, O. A. Ladyzhenskaja, Ed., Amer. Math. Soc., Providence, RI (1965). MR 35:1965

18. J. L. Vazquez, The Free Boundary Problem for the Heat Equation with fixed Gradient Condition, Proc. Internat. Conf. "Free Boundary Problems, Theory and Applications", Zakopane, Poland, Pitman Research Notes in Mathematics 363, pp. 277-302, 1995. MR 98h:35246

Division of Mathematics and Computer Science, Free University Amsterdam, De Boelelaan 1081, 1081 HV Amsterdam, The Netherlands

E-mail address: olivier@cs.vu.nl

Dipartimento di Matematica, Università di Parma, Via D’Azeglio 85/A, 43100 Parma, ITALY

E-mail address: lunardi@unipr.it

$U R L:$ http://math.unipr.it/ lunardi 